\title{
Tratamiento quirúrgico periimplantario
}

\section{Surgical treatment peri-implantitis}

\author{
ORTIZ-VIGÓN CARNICERO A* \\ FABRIZI $S *$ \\ BASCONES MARTÍNEZ A**
}

\begin{abstract}
Ortiz-Vigón Carnicero A, Fabrizi S, Bascones Martínez A. Tratamiento quirúrgico periimplantario. Av Periodon Implantol. 2012; 24, 1: 11-17.
\end{abstract}

\begin{abstract}
RESUMEN
Uno de los factores clave del éxito a largo plazo de los implantes dentales es el mantenimiento de la salud de los tejidos entorno a éstos. El biofilm bacteriano es capaz de inducir alteraciones inflamatorias en los tejidos blandos periimplantarios, lo que puede llevar a la destrucción de los mismos, produciendo finalmente el fracaso del implante. Para la prevención de esta patología se han propuesto múltiples estrategias terapéuticas, aunque no se ha evidenciado cual es la más efectiva. Objetivos: Identificar el protocolo terapéutico más efectivo para el tratamiento de la patología periimplantaria en implantes osteointegrados.

Conclusión: La cirugía de acceso ha demostrado resolver un 58\% de los casos de periimplantitis. Ninguno de los métodos de descontaminación de superficies ha demostrado ser superior a las demás.
\end{abstract}

PALABRAS CLAVE: Periimplantitis, tratamiento periimplantario, tratamiento quirúrgico periimplantario.

\section{SUIMIMARY}

One of the key factors for the long-term success of oral implants is the maintenance of healthy tissues around them. Bacterial plaque accumulation induces inflammatory changes in the soft tissues surrounding oral implants and it may lead to their progressive destruction (perimplantitis) and ultimately to implant failure. Different treatment strategies for perimplantitis have been suggested, however it is unclear which are the most effective.

Objectives: To identify the most effective interventions for treating perimplantitis around osseointegrated dental implants.

Conclusion: Access surgery has demonstrate that resolution occurred in $58 \%$ of the lesions. No single method of surface decontamination was found to be superior.

KEY WORDS: Peri-implantitis, peri-implantitis treatment, surgical treatment peri-implantitis.

Fecha de recepción: 2 de septiembre de 2010.

Fecha de aceptación: 15 de octubre de 2010.

\section{INTRODUCCIÓN}

Tradicionalmente los dientes perdidos por diversas causas se han repuesto mediante la colocación de prótesis fijas o removibles, permitiendo la restauración de la función masticatoria, fonética y estética. En 1977,
Brånemark presentó su investigación a 10 años, demostrando que el hueso puede crecer íntimamente unido a la superficie de un implante de titanio (Brånemark 1977).

Pese a los excelentes resultados del tratamiento mediante implantes osteointegrados y su supervivencia

\footnotetext{
* Odontólogo. Alumno Máster de Periodoncia. Facultad de Odontología. Universidad Complutense de Madrid.

** Catedrático de Medicina Bucal y Periodoncia. Departamento de Medicina y Cirugía Bucofacial (Estomatología III). Facultad de Odontología. Universidad Complutense de Madrid. España.
} 
a largo plazo (Berglundh et al. 2002) es innegable la existencia, y más aún, el aumento de la incidencia de la patología periimplantaria.

La periimplantitis se define como una reacción inflamatoria con perdida de soporte entorno a un implante (Albrektsson et al. 1994, Lindhe and Meyle, 2008). La frecuencia de la periimplantitis se encuentra en un rango de entre un 5-8\% (Berglundh et al. 2002) y con resultados similares, de hasta el $16 \%$, en otros estudios más recientes de seguimiento a largo plazo (RoosJansaker et al.2006). En una revisión, se concluye que el tratamiento no quirúrgico puede resolver o estabilizar las lesiones periimplantarias a corto plazo (RoosJansaker et al. 2003). Aunque la pérdida ósea y por tanto la exposición de las espiras con superficies habitualmente rugosas, hace muy compleja la descontaminación de las superficies mediante terapias no quirúrgicas (Kreisler et al. 2005, Schwarz et al. 2006a). Las superficies de los implantes han evolucionado en las últimas 2 décadas, pasando de superficies maquinadas, lisas, de titanio a superficies rugosas. Las superficies rugosas tienen la ventaja de aumentar la estabilidad del implante en el hueso y además muestran un mayor índice de BIC (Bone to Implant Contact), pero a su vez al exponerse al medio oral facilitan la colonización bacteriana y la formación del biofilm teniendo como resultado la patología periimplantaria (Miller, 2004). La incidencia de la periimplantitis en implantes de superficie rugosa es mayor como concluye una revisión sistemática de Cochrane (Espósito et al. 2007), observando que tras 3 años de carga hay una prevalencia significativamente mayor de patología periimplantaria en implantes con superficie rugosa verus implantes maquinados.

Por otra parte numerosos estudios han resaltado que los pacientes con un historial previo de periodontitis crónica $(28,6 \%)$ tienen un riesgo significativamente mayor de padecer periimplantitis que los sanos $(5,8 \%)$ (Karoussis et al. 2003). Esta asociación ha sido posteriormente sostenida por otros estudios (Karoussis et al. 2004, Karoussis et al. 2003). Dos recientes revisiones sistemáticas (Schou et al. 2006, Karoussis et al.2007) concluyen que los implantes colocados en pacientes diagnosticados de periodontitis crónica muestran un incidencia mayor que aquellos colocados en pacientes sanos, afirmando así, que la periodontitis crónica es un factor predisponente del desarrollo de la periimplantitis (Renvert and Persson, 2009).

Para el tratamiento de la periimplantitis se han sugerido múltiples terapias, a menudo combinadas, entre las cuales cabe destacar: el desbridamiento mecánico, terapia antiséptica local y/o sistémica y los distintos protocolos quirúrgicos, con el objetivo de reducir - eliminar la carga bacteriana, reducir la rugosidad del implante y detoxificar su superficie mediante diferentes técnicas. Tras alcanzar fin primario de la cirugía que no es otro que la obtención de una superficie implantaria libre de bacterias es necesario valorar la necesidad de corregir las estructuras anatómicas para mejorar el control de placa y además obtener un resultado estético. Esto se puede conseguir mediante cirugía resectiva o bien mediante procedimientos regenerativos incluyendo la regeneración ósea guiada y los injertos óseos (Roos-Jansaker et al. 2003; Schou et al. 2004)

Por lo tanto el objetivo último es la resolución de la patología periimplantaria que además debería considerar la regeneración de los tejidos perdidos y el restablecimiento de la osteointegración a lo largo de la superficie previamente contaminada del implante (Schou et al.2004). Para lograr este objetivo se han propuesto múltiples terapias, como el desbridamiento mecánico, el uso de antisépticos, antibióticos, la cirugía periimplantaria e incluso la explantación del implante en casos de mal pronóstico (Mombelli and Lang, 1998).

\section{OBJETIVOS}

El objetivo de esta revisión, es analizar la evidencia disponible a cerca de la eficacia de los distintos protocolos terapéuticos quirúrgicos para el tratamiento de la periimplantitis.

\section{TRATAMIENTO QUIRÚRGICO PERIIMPLANTARIO VERSUS NO QUIRÚRGICO}

Antes de analizar los resultados de la terapia quirúrgica periimplantaria conviene comparar brevemente esta opción terapéutica con la no quirúrgica. En un estudio a boca partida en modelo animal (perros) resalta el hecho de la significativa superioridad del desbridamiento abierto en relación al cerrado (Schwarz et al. 2006b). A los 3 meses ambos grupos mostraron una mejora significativa de los parámetros clínicos, pero únicamente el grupo de desbridamiento abierto mostró una mejora significativa de los parámetros radiográficos. Además, el análisis histológico mostró una diferencia significativa de reosteointegra- 
ción de $1 \%$ del no quirúrgico y un $44 \%$ del grupo quirúrgico.

En relación a los parámetros microbiológicos el colgajo de acceso junto con irrigación con clorhexidina muestra una mayor reducción (Hayek et al. 2005) de periodontopatógenos en comparación con el raspado no quirúrgico en combinación con terapia fotodinámica.

\section{DESCONTAMINACIÓN DE LA SUPERFICIE DEL IMPLANTE}

Parece establecido que la obtención de una superficie implantaria conductiva es un requisito indispensable para el éxito de un tratamiento regenerativo periimplantario (Schwartz et al. 1997). Los contaminantes como las bacterias y sus productos metabólicos, el cálculo, y las células que dan origen al tejido blando deben ser eliminadas sin alterar, en la medida de lo posible, la superficie del implante. En cualquier caso, sigue siendo incierto el umbral de erradicación de estos contaminantes a partir del cual es posible alcanzar un resultado exitoso (Mombelli, 2002).

Numerosos estudios sugieren diferentes métodos de descontaminación de la superficie implantaria en combinación con el tratamiento quirúrgico periimplantario (Hammerle et al. 1995, Klinge et al. 2002). Algunos de los métodos de descontaminación propuestos son inadecuados si el objetivo terapéutico es la reosteointegración del implante y no solo la eliminación de la patología periimplantaria (Speelman et al. 1992). Dentro de estos métodos poco recomendables cabe destacar, el uso de curetas de acero, puntas convencionales de ultrasonidos y algunas puntas de láser, que pueden alterar gravemente la superficie del implante, interfiriendo en la regeneración ósea periimplantaria. Aunque dicha alteración puede ser prevenida mediante el uso de puntas de ultrasonidos y curetas de resina, de fibra de carbono o de teflón, la exposición al medio oral de una superficie implantaria rugosa conlleva una dificulta añadida para la descontaminación versus una superficie maquinada o pulida.

Varios estudios que analizan en modelo animal diferentes técnicas de descontaminación de la superficie periimplantaria en combinación con la cirugía no hallan diferencias en relación al resultado del tratamiento (Persson et al. 1999, Deppe et al. 2001, Schou et al. 2003 , You et al.2007). No hallan diferencias en cuanto a la reosteointegración entre el uso de un algodón im- pregnado en suero salino y un cepillo con una pasta abrasiva (Persson et al. 1999) ni entre el láser de dióxido de carbono y/o un spray abrasivo durante la cirugía (Deppe et al. 2007). Otro estudio compara 4 protocolos distintos de descontaminación quirúrgica:

1. Spray abrasivo seguido de ácido cítrico,

2. Spray abrasivo,

3. Gasa impregnada en ácido cítrico y

4. Gasa con clorhexidina al 0,1\% y suero salino alternados (Schou et al. 2003).

Todos los defectos periimplantarios son rellenados por hueso autólogo y cubiertos por una membrana de ePTFE. No hallando diferencias significativas ni en los parámetros clínicos, ni en los radiográficos, ni en los histológicos. En base a la evidencia los autores concluyen que se puede descontaminar de forma eficaz la superficie de implantes rugosos mediante la aplicación de una gasa embebida en clorhexidina durante la cirugía periimplantaria además de hallar una mayor tasa de reosteointegración en implantes de superficie rugosa, SLA (You et al. 2007, Persson et al. 2005) y una menor tasa de reosteointegración sin regeneración ósea guiada (You et al. 2007).

Además de los métodos mecánicos y químicos cabría mencionar las técnicas fotodinámicas de descontaminación implantaria. En este estudio se compara el desbridamiento quirúrgico utilizando el láser Er:YAG, un ultrasonidos y curetas de plástico en combinación con gel de metronidazol para la descontaminación del implante (Schwarz et al. 2006a). Aunque clínicamente el ultrasonidos obtenía los mejores resultados, histológicamente el láser obtuvo el mayor grado de reosteointegración con un $44,8 \%$ versus un $8,7 \%$ del ultrasonidos y un $14,8 \%$ de las curetas de plástico junto con metronidazol.

De forma general, la mayoría de los estudios analizados obtienen una resolución de la lesión inflamatoria, pero no obtienen una significativa reosteointegración de las superficies previamente contaminadas (Claffey et al. 2008). Los resultados histológicos demuestran una encapsulación de tejido conectivo separando el implante del hueso adyacente (Grunder et al. 1993, Persson et al. 1999, Persson et al. 2004, Wetzel et al. 1999).

En conclusión, la resolución de la patología periimplantaria puede alcanzarse mediante diversos métodos de descontaminación de la superficie del implante. Aunque la evidencia indica que el desbridamiento a colgajo abierto no obtiene una significativa reosteointegración aunque sí puede obtener un relleno óseo. Por 
otra parte implantes descontaminado y colocados en lechos nuevos son capaces de obtener una elevada tasa de reosteointegración (Kolonidis et al. 2003, Alhag et al. 2008) este hecho hace resaltar que hay otros factores, además de la descontaminación del implante, que influyen en el éxito del tratamiento.

\section{INFLUENCIA DE LA SUPERFICIE DEL IMPLANTE}

Diversos estudios han analizado entre sus variables la influencia de la superficie implantaria en relación al éxito del tratamiento. (Shibli et al. 2003) resalta la influencia de las diferentes superficies en la regeneración ósea y en la reosteointegración, utilizando membranas de politetrafluoroetileno expandido (e-PTFE) hallando la mayor evidencia de regeneración asociada a los implantes de superficie HA-coated (hidroxiapatita) y la menor con la superficie de titanio pura (cpTI). En relación a la reosteointegración los mejores resultados los obtienen las superficies TPS (titanium plasma sprayed). Otros estudios que analizan la influencia de la superficie son los de (Wetzel et al. 1999) que halla diferencias significativas en términos de relleno óseo y reosteointegración entre la SLA (sand blasted large grit acid etched) siendo la mejor y seguida de la TPS (titanium plasma sprayed) y por último la maquinada. Estos resultados están en concordancia con los obtenidos por (Sennerby et al. 2005, Persson et al. 2004) ambos demuestran la superioridad de la superficie SLA.

\section{TRATAMIENTO QUIRÚRGICO PERIIMPLANTARIO}

En una serie de casos publicada en 2003 se evalúa el resultado a largo plazo de la cirugía de acceso junto con antibióticos sistémicos y descontaminación con peróxido de hidrógeno. Los resultados de este estudio muestran una resolución de la patología periimplantaria en el 58\% de los implantes. Pese al tratamiento recibido 7 de los 26 implantes tratados se pierden y hay una progresión de la pérdida de inserción periimplantaria en 4 implantes más (Leonhardt et al. 2003).

Otro estudio comparativo analiza las diferencias entre modificar o no la superficie del implante junto con la cirugía de reposición apical. La implantoplastia debe ser considerada como protocolo coadyuvante en caso de cirugía periimplantaria resectiva (Romeo et al. 2007, Romeo et al. 2005). 19 pacientes tratados de periim- plantitis reciben antibióticos sistémicos y cirugía resectiva periimplantaria, a 10 de los cuales se les realiza implantoplastia de la superficie implantaria supraósea. Los registros radiográficos muestran en el grupo de implantoplastia una significativa mejoría ósea en comparación con el grupo de colgajo de reposición apical.

\section{LÁSER COMO TRATAMIENTO COADYUVANTE AL TRATAMIENTO QUIRÚRGICO PERIIMPLANTARIO}

Múltiples estudios han analizado el efecto de las terapias fotosensibilizadoras en el tratamiento de las superficies implantarias. (Dortbudak et al. 2001) analiza en potencial de la terapia fotosensibilizadora in vivo. Miden los niveles de Aggregatibacter Actinomycetemcomitans Aa, Porphyromonas gingivalis Pg y Prevotella intermedia $P i$ antes y después de la aplicación de un colorante y tras la aplicación del láser. Los resultados muestran que aunque no se obtiene la erradicación completa de las 3 especies si se produce una reducción estadísticamente significativa de las $3(p<0,0001)$ en relación a los niveles iniciales.

En una serie de casos se utiliza para la descontaminación del implante un láser de baja intensidad antes de rellenar el defecto óseo con hueso autólogo y cubrir el defecto con una membrana de e-PTFE. Los resultados a corto plazo corroboran la eficacia de las terapias fotoactivas (Haas et al. 2000).

En una investigación más reciente se compara la terapia de descontaminación convencional comparada con el tratamiento con láser de $\mathrm{CO}_{2}$. Tras el análisis de los resultados se puede concluir que el láser tiene un efecto significativamente mejor a corto plazo aunque estos resultados no se mantienen a largo plazo (Deppe et al. 2007).

(Takasaki et al. 2007) obtiene unos resultados del $69,7 \%$ de reosteointegración utilizando un láser ErYAG para la descontaminación de la superficie periimplantaria y para la eliminación del tejido de granulación.

De los estudios analizados se puede concluir que el tratamiento periimplantario combinando la cirugía de acceso con el láser puede obtener mejores resultados que el tratamiento convencional solo. Aún así son necesarios más estudios para confirmar estos resultados (Renvert et al. 2009). 


\section{CONCLUSIONES}

\section{Implicaciones prácticas:}

- El desbridamiento a colgajo con descontaminación de la superficie es significativamente más efectivo que el desbridamiento periimplantario sin cirugía en bolsas $\geq 5 \mathrm{~mm}$.

- El desbridamiento a colgajo con descontaminación de la superficie resuelve la patología periimplantaria, promueve el relleno óseo y permite la reosteointegración.

- La reosteointegración es un resultado reproducible y más frecuentemente vinculada a superficies implantaria tratadas o de mayor rugosidad, en comparación con las superficies mecanizadas o pulidas.

- No hay un solo método de descontaminación de superficies, utilizado de forma individual, que sea superior a los demás en relación a los resultados clínicos a largo plazo.

- No hay evidencia suficiente para afirmar la necesidad del uso coadyuvante de antibióticos sistémicos en combinación con el tratamiento periimplantario.

\section{BIBLIOGRAFÍA}

1. Albrektsson T, Isidor F. Consensus report of session IV. In: Lang NP, Karring T. (eds). Proceedings of the First European Workshop on Periodontology 1994:365-9. London: Quintessence.

2. Albrektsson TO, Johansson CB, Sennerby L. Biological aspects of implant dentistry: osseointegration. Periodontol 2000, 1994;4:58-73.

3. Alhag M, Renvert S, Polyzois I, Claffey N. (2008). Reosseointegration on rough implant surfaces previously coated with bacterial biofilm: an experimental study in the dog. Clin Oral Implants Res 2008;19:182-7.

4. Berglundh T, Persson $\mathrm{L}$, Klinge B. A systematic review of the incidence of biological and technical complications in implant dentistry reported in prospective longitudinal studies of at least 5 years. J Clin Periodontol 2002; 29(Suppl 3):197-212; discussion 232-193.

5. Brånemark PI, Hansson BO, Adell R, Breine U, Lindström J, Hallén O, Ohman A. Osseointegrated implants in the treatment of the edentulous jaw. Experience from a 10year period. Scand J Plast Reconstr Surg 1977;16(Suppl.):1132.
6. Claffey N, Clarke E, Polyzois I, Renvert S. Surgical treatment of peri-implantitis. J Clin Periodontol 2008;35: 316-32.

7. Deppe H, Horch HH, Henke J, Donath K. Per-implant care of ailing implants with the carbon dioxide laser. Int J Oral Maxillofac Implants 2001;16:659-67.

8. Deppe $\mathrm{H}$, Horch $\mathrm{HH}, \mathrm{Neff}$ A. Conventional versus $\mathrm{CO}_{2}$ laser-assisted treatment of peri-implant defects with the concomitant use of pure-phase beta-tricalcium phosphate: a 5-year clinical report. Int J Oral Maxillofac Implants 2007; 22:79-86.

9. Dortbudak O, Haas R, Bernhart T, Mailath-Pokorny G. Lethal photosensitization for decontamination of implant surfaces in the treatment of peri-implantitis. Clin Oral Implants Res 2001;12:104-8.

10. Espósito M, Murray-Curtis L, Grusovin MG, Coulthard $\mathrm{P}$, Worthington HV. Interventions for replacing missing teeth: different types of dental implants. 2007. Cochrane Database Syst Rev, CD003815.

11. Grunder U, Hurzeler MB, Schupbach P, Strub JR. Treatment of ligature-induced peri-implantitis using guided tissue regeneration: a clinical and histologic study in the beagle dog. Int J Oral Maxillofac Implants 1993;8:282-93.

12. Haas R, Baron M, Dortbudak O, Watzek G. Lethal photosensitization, autogenous bone, and e-PTFE membrane for the treatment of peri-implantitis:preliminary results. Int J Oral Maxillofac Implants 2000;15: 374-82.

13. Hammerle CH, Fourmousis I, Winkler JR, Weigel C, Bragger U, Lang NP. Successful bone fill in late periimplant defects using guided tissue regeneration. A short communication. J Periodontol 1995;66:303-8.

14. Hayek RR, Araujo NS, Gioso MA, Ferreira J, BaptistaSobrinho CA, Yamada AM, Ribeiro MS. Comparative study between the effects of photodynamic therapy and conventional therapy on microbial reduction in ligatureinduced peri-implantitis in dogs. J Periodontol 2005;76: 1275-81.

15. Karoussis IK, Kotsovilis S, Fourmousis I. A comprehensive and critical review of dental implant prognosis in periodontally compromised partially edentulous patients. Clin Oral Implants Res 2007;18:669-79.

16. Karoussis IK, Muller S, Salvi GE, Heitz-Mayfield LJ, Bragger U, Lang NP. Association between periodontal 
and peri-implant conditions: a 10-year prospective study. Clin Oral Implants Res 2004;15:1-7.

17. Karoussis IK, Salvi GE, Heitz-Mayfield LJ, Bragger U, Hammerle CH, Lang NP. Long-term implant prognosis in patients with and without a history of chronic periodontitis: a 10-year prospective cohort study of the ITI Dental Implant System. Clin Oral Implants Res 2003; 14:329-39.

18. Klinge B, Gustafsson A, Berglundh T. A systematic review of the effect of anti-infective therapy in the treatment of peri-implantitis. J Clin Periodontol 2002;29(Suppl 3): 213-25.

19. Kolonidis SG, Renvert S, Hammerle CH, Lang NP, Harris D, Claffey N. Osseointegration on implant surfaces previously contaminated with plaque. An experimental study in the dog. Clin Oral Implants Res 2003; 14:37380.

20. Kreisler M, Kohnen W, Christoffers AB, Gotz H, Jansen B, Duschner $H$, d'Hoedt $B$. In vitro evaluation of the biocompatibility of contaminated implant surfaces treated with an Er:YAG laser and an air powder system. Clin Oral Implants Res 2005;16:36-43.

21. Leonhardt A, Dahlen G, Renvert S. Five-year clinical, microbiological, and radiological outcome following treatment of peri-implantitis in man. J Periodontol 2003; 74:1415-22.

22. Lindhe J, Meyle J. Peri-implant diseases: Consensus Report of the Sixth European Workshop on Periodontology. J Clin Periodontol 2008;35:282-5.

23. Miller RJ.Treatment of the contaminated implant surface using the Er,Cr:YSGG laser. Implant Dent 2004;13:16570.

24. Mombelli A. Microbiology and antimicrobial therapy of peri-implantitis. Periodontol 2000, 2002;28:177-89.

25. Mombelli A, Lang NP. The diagnosis and treatment of peri-implantitis. Periodontol 2000, 1998;17:63-76.

26. Persson LG, Araujo MG, Berglundh T, Grondahl K, Lindhe J. Resolution of peri-implantitis following treatment. An experimental study in the dog. Clin Oral Implants Res 1999;10:195-203.

27. Persson LG, Mouhyi J, Berglundh T, Sennerby L, Lindhe J. Carbon dioxide laser and hydrogen peroxide conditioning in the treatment of periimplantitis: an experimental study in the dog. Clin Implant Dent Relat Res 2004;6: 230-8.

28. Persson M, Svenarud P, Flock JI, van der Linden J. Carbon dioxide inhibits the growth rate of Staphylococcus aureus at body temperature. Surg Endosc 2005;19: 91-4.

29. Renvert S, Persson GR. Periodontitis as a potential risk factor for peri-implantitis. J Clin Periodontol 2009;36 (Suppl 10):9-14.

30. Renvert S, Polyzois I, Maguire R. Re-osseointegration on previously contaminated surfaces: a systematic review. Clin Oral Implants Res 2009;20(Suppl 4):216-27.

31. Romeo E, Ghisolfi M, Murgolo N, Chiapasco M, Lops D, Vogel G. Therapy of peri-implantitis with resective surgery. A 3-year clinical trial on rough screw-shaped oral implants. Part I: clinical outcome. Clin Oral Implants Res 2005;16:9-18.

32. Romeo E, Lops D, Chiapasco M, Ghisolfi M, Vogel G. Therapy of peri-implantitis with resective surgery. A 3year clinical trial on rough screw-shaped oral implants. Part II: radiographic outcome. Clin Oral Implants Res 2007;18:179-87.

33. Roos-Jansaker AM, Lindahl C, Renvert H, Renvert S. Nineto fourteen-year follow-up of implant treatment. Part I: implant loss and associations to various factors. J Clin Periodontol 2006;33:283-9.

34. Roos-Jansaker AM, Renvert S, Egelberg J.Treatment of periimplant infections: a literature review. J Clin Periodontol 2003;30:467-85.

35. Schou S, Berglundh T, Lang NP. Surgical treatment of peri-implantitis. Int J Oral Maxillofac Implants 2004;19 (Suppl):140-9.

36. Schou S, Holmstrup P, Jorgensen T, Skovgaard LT, Stoltze $\mathrm{K}$, Hjorting-Hansen $\mathrm{E}$, Wenzel $\mathrm{A}$. Implant surface preparation in the surgical treatment of experimental peri-implantitis with autogenous bone graft and ePTFE membrane in cynomolgus monkeys. Clin Oral Implants Res 2003;14:412-22.

37. Schou S, Holmstrup P, Worthington HV, Espósito M. Outcome of implant therapy in patients with previous tooth loss due to periodontitis. Clin Oral Implants Res 2006;17(Suppl 2):104-123. 
38. Schwartz Z, Kieswetter K, Dean DD, Boyan BD. Underlying mechanisms at the bone-surface interface during regeneration. J Periodontal Res 1997;32: 16671 .

39. Schwarz, F, Bieling, K, Nuesry, E, Sculean, A, Becker, J. Clinical and histological healing pattern of periimplantitis lesions following non-surgical treatment with an Er:YAG laser. Lasers Surg Med 2006a;38: 66371 .

40. Schwarz F, Jepsen S, Herten M, Sager M, Rothamel D, Becker J. Influence of different treatment approaches on non-submerged and submerged healing of ligature induced peri-implantitis lesions: an experimental study in dogs. J Clin Periodontol 2006b;33:584-95.

41. Sennerby L, Persson LG, Berglundh T, Wennerberg A, Lindhe J. Implant stability during initiation and resolution of experimental periimplantitis: an experimental study in the dog. Clin Implant Dent Relat Res 2005; 7:136-40.

42. Shibli JA, Martins MC, Nociti FH, Jr, García VG, Marcantonio E, Jr. Treatment of ligature-induced periimplantitis by lethal photosensitization and guided bone regeneration: a preliminary histologic study in dogs. J Periodontol 2003;74:338-45.
43. Speelman JA, Collaert B, Klinge B. Evaluation of different methods to clean titanium abutments. A scanning electron microscopic study. Clin Oral Implants Res 1992; 3:120-7.

44. Takasaki AA, Aoki A, Mizutani K, Kikuchi S, Oda S, Ishikawa I. Er:YAG laser therapy for peri-implant infection: a histological study. Lasers Med Sci 2007;22:143-57.

45. Wetzel AC, Vlassis J, Caffesse RG, Hammerle CH, Lang NP. Attempts to obtain re-osseointegration following experimental peri-implantitis in dogs. Clin Oral Implants Res 1999;10:111-9.

46. You TM, Choi BH, Zhu SJ, Jung JH, Lee SH, Huh JY, Lee HJ, Li J. Treatment of experimental peri-implantitis using autogenous bone grafts and platelet-enriched fibrin glue in dogs. Oral Surg Oral Med Oral Pathol Oral Radiol Endod 2007;103:34-7.

\section{CORRESPONDENCIA}

\section{A. Ortiz-Vigón Carnicero}

Facultad de Odontología

Universidad Complutense de Madrid

Plaza Ramón y Cajal

28040 Madrid

Correo electrónico: clinicaortizvigon@gmail.com 